\title{
CHAOTIC DATA ENCRYPTION FOR LONG-DISTANCE MONITORING OF NUCLEAR REACTORS
}

\author{
Günyaz Ablay \\ The Ohio State University, Nuclear Engineering Program, Columbus, OH 43210, USA \\ E-mail: ablay.1@osu.edu \\ Phone: 6143709776
}

\begin{abstract}
Can Emre Koksal
The Ohio State University, Department of Electrical and Computer Engineering, Columbus, OH 43210, USA
\end{abstract}

Tunc Aldemir

The Ohio State University, Nuclear Engineering Program, Columbus, OH 43210, USA

Total Number of Pages: 41

Number of Tables: 2

Number of Figures: 11 


\title{
CHAOTIC DATA ENCRYPTION FOR LONG-DISTANCE MONITORING OF NUCLEAR REACTORS
}

\author{
Günyaz Ablay ${ }^{1}$, C. Emre Koksal ${ }^{2}$, and Tunc Aldemir ${ }^{3}$ \\ ${ }^{1,3}$ Nuclear Engineering Program \\ ${ }^{2}$ Department of Electrical and Computer Engineering \\ The Ohio State University, Columbus, OH 43210, USA \\ ${ }^{l}$ E-mail: ablay.1@osu.edu
}

\begin{abstract}
A secure long-distance monitoring scheme is proposed for nuclear engineering applications using chaos synchronization and nonlinear observers for on-line transmittal of operational data, distance monitoring, fault detection and other related processes. The proposed system consists of three components: a) chaotic transmitter to encrypt and send signals coming from a message originating system, b) chaotic receiver to decrypt information signals, and c) reconstruction of the message originating system using the decrypted signals. The Lorenz chaotic system whose parameters are defined as nonlinear functions of the state variables to improve the security level of the chaosbased communication is considered as chaotic encrypter. In the receiver section, a nonlinear observer is used to provide synchronization and to decrypt message signal. A similar nonlinear observer is employed to reconstruct the message originating system state variables from the recovered message signal. Numerical results and case studies against certain passive eavesdropping attacks are provided to demonstrate the resilience of the proposed method. A reduced order boiling water reactor model is used as the message originating system in the illustrations.
\end{abstract}




\section{NOMENCLATURE}

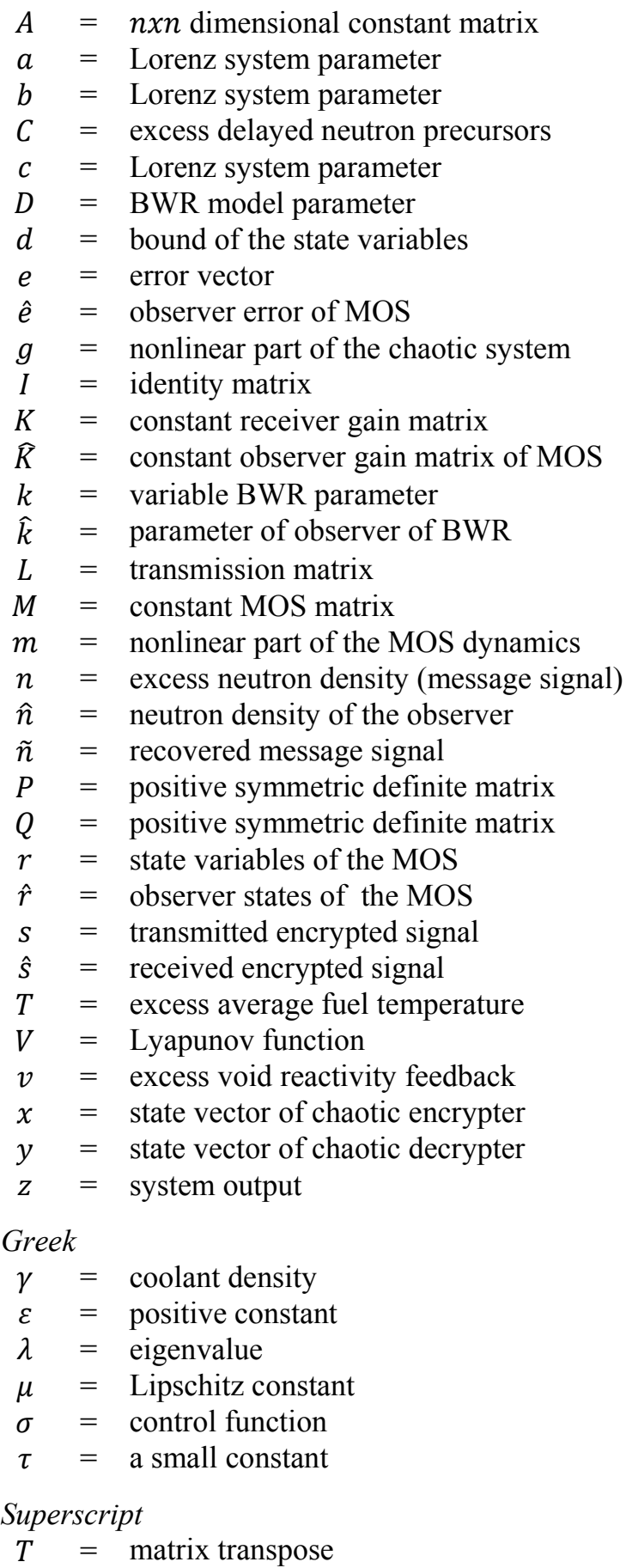




\section{INTRODUCTION}

Secure long-distance monitoring of plant data is becoming increasingly important needs for safe and efficient operation of nuclear power plants. In a recent workshop on Advanced Instrumentation, Information, and Control Systems and Human-System Interface Technologies, the development of an automated system that compares transient signatures to occurrences at other plants by automatically searching and analyzing a database of events and responses was identified as a desired capability that could improve plant operations. ${ }^{1}$ Small, modular reactors designed to operate in 8-10 unit energy parks (Ref. 2) are expected to rely on centralized control. Future mixed nuclear parks, where fast reactors would be deployed to gain fuel sustainability with high temperature reactors providing the means to transmute the minor actinides (Ref. 3) would require secure and efficient data exchange between different units. Long-distance monitoring can facilitate evaluation, research, fault detection, data storage, and even can give rise to remote control of the reactors. Similar arguments can be made for space nuclear reactors and the idea of deep underground parks. ${ }^{4}$ For both wired and wireless applications, communication security is an important concern.

Recently many chaos-based communication methods have been proposed to achieve security in communication. Chaotic systems are known to have high parameter and initial condition sensitivities, leading to a significant unpredictability, and thus making them an attractive tool for encryption. ${ }^{5-6}$ In chaotic cryptosystems, nonlinear encryption methods are used to encrypt the secure message at the transmitter side. The original message can be recovered at the receiver side with an inverse transformation if chaos synchronization is achieved. Among the publications dedicated to chaos synchronization, many different approaches can be found, 
including nonlinear control, ${ }^{7}$ nonlinear backstepping control, ${ }^{8}$ synchronization in time-delayed systems, ${ }^{9}$ active control, ${ }^{10}$ observer-based approach, ${ }^{11}$ feedback controllers ${ }^{12}$ and adaptive control $^{13}$. Some publications dedicated to chaotic cryptography by using chaos synchronization can be found in References 14-21.

It has been pointed out that some chaotic encryption algorithms (e.g. References 14-18) may have vulnerability due to the use of constant parameter values in the transmitting and receiving chaotic systems. In References 22-32, it is shown that several chaos-based cryptosystems can be broken systematically through optimization processes, low pass filtering and high pass filtering under the assumption that the structure of the chaotic decrypter is known. References 31-32 propose some requirements for security of chaos based communications. The main requirements are given as the usage of bounded nonlinear functions as keys instead of constant parameter values and the usage of multi-system encryption schemes, ${ }^{31}$ namely, using several different chaotic systems in order to cause the chaotic signal to become even more complex. The message signal processed in more than two different chaotic systems is claimed to lead to unreasonable amount of computational effort for decryption, at least for computer-based algorithms. $^{20}$

As it is true for all process systems, nuclear reactor observables contain noisy data. The above methods are inherently non-robust with respect to noisy observations. In this paper, a robust chaos synchronization method is proposed for long-distance monitoring of nuclear power plants with variable transmitter system parameter values as keys. The method is illustrated using a reduced order boiling water reactor (BWR) model as an example system. The contribution of this paper is threefold: 
- The chaotic transmitter system parameters are defined as nonlinear functions of the state variables of the transmitter system as private keys instead of linear functions of the parameters as proposed in References 19-21, which increases the difficulty for an eavesdropper to decrypt the transmitted signals.

- A nonlinear observer is designed for synchronization of transmitter and receiver chaotic systems to accommodate for the nonlinearities in the systems and the variability in the transmitting system parameters due to their chaotic nature arising from their definition as nonlinear functions of the state variables of the transmitter system.

- It is shown that the message originating system (MOS) can be reconstructed and tracked from the recovered message signal using a similar nonlinear observer even if MOS behavior is highly nonlinear (and possibly chaotic) with noisy output and parameter uncertainty.

The use of the nonlinear observer is a particularly significant contribution because reconstruction and tracking of the MOS can be very sensitive to how well the received data represent the actual MOS dynamics.

The paper is organized as follows. A brief mathematical description of the problem is presented in Sec. II. The example MOS model is given in Sec. III. An application and numerical simulations are given in Sec. IV to demonstrate the effectiveness of the proposed method. Some basic security analyses of the proposed chaos-based communication method against passive eavesdroppers are given in Sec. V. Finally, the conclusions are given in Sec. VI. 


\section{PROBLEM DESCRIPTION}

In chaotic masking, a chaotic signal hides the information signal in the transmitter module and the hidden information is recovered in the receiver module. ${ }^{31}$ The procedure for secure communication (against passive eavesdroppers) and long distance monitoring consists of three parts: a) encryption of MOS information, b) decryption of the MOS information, and, c) reconstruction of the MOS, as illustrated graphically in Fig. 1 for a 3rd order chaotic encrypter. The reconstruction of MOS (or its acceptable fidelity equivalent) is needed to gain a better understanding of the plant state and the relative relationships of plant variables which then can be used for both diagnostic and prognostic purposes. In the transmitter section, a chaotic system is used as the encrypter. Some of the state variables of the modulator are sent to the receiver via public channels to rebuild the state variables (synchronization) of the chaotic encrypter at the

receiver. ${ }^{14-32}$ In our chaotic encrypter, one state variable $\left(x_{1}\right)$ is used for synchronization and the chaotic masking modulation is applied by using the other two state variables $\left(x_{2}\right.$ and $\left.x_{3}\right)$ of the chaotic encrypter. The nonlinear parameters of the chaotic decrypter (see Sec. IV) are assumed to be privately exchanged by the transmitter and the receiver, and are unknown by the eavesdropper. The message signal $n$ is masked by the chaotic signals generated by the encrypter as will be explained in Sec. II.A, and the encrypted message signal $s$ is sent to the receiver via the public channel. In the receiver section, a chaotic decrypter matched to the chaotic encrypter is used as an observer to obtain chaos synchronization between encrypter and decrypter. There, the encrypted message signal $s$ is decrypted by inverse transformation $s^{-1}$ to obtain a close replica $\tilde{n}$ of the message signal $n$. For the reconstruction of the MOS, we use another nonlinear observer to rebuild the MOS states for tracking by applying the recovered message signal $\tilde{n}$ to this observer. The eavesdropper given in Fig.1 is assumed to have access 
only to the information transmitted over the public channel. Some basic security analyses of the proposed method will be described in Sec. V.

Figure 1

The mathematical structure of the proposed approach given in Fig. 1 is described in Sections II.A through II.C.

\section{II.A. Transmitter Section}

Consider the following system as a chaotic encrypter

$$
\dot{\boldsymbol{x}}=A \boldsymbol{x}+\boldsymbol{g}(\boldsymbol{x}) \text { and } \quad z=C \boldsymbol{x}
$$

where

$x \in R^{3}=$ chaotic encrypter state vector such that $\|x\|<d_{1}$ for some $d_{1}>0$,

$A \in R^{3 x 3}=$ constant matrix associated with the linear part of the chaotic system,

$z \in R=$ synchronization signal observed at the output,

$C \in R^{1 \times 3}=$ output matrix, i.e. $C=[1,0,0]$ since $x_{1}$ is selected as a synchronization signal,

$\boldsymbol{g}: R^{3} \rightarrow R^{3}=$ nonlinear part of the chaotic system which is assumed to satisfy the Lipschitz condition with a Lipschitz constant $\mu_{1}>0$, i.e. for all $\boldsymbol{x}$ and $\boldsymbol{y}$

$$
\|\boldsymbol{g}(\boldsymbol{y})-\boldsymbol{g}(\boldsymbol{x})\| \leq \mu_{1}\|\boldsymbol{y}-\boldsymbol{x}\| .
$$

As indicated earlier and shown in Fig. 1, two signals will be sent to the receiver: a) synchronization signal $x_{1}$ to be used to rebuild the state variables of the chaotic encrypter at the receiver, and b) encrypted message signal:

$$
s=n \tau+x_{2}+x_{3},
$$


where

$n=$ message signal with additive noise,

$\tau=$ a constant to attenuate the message signal.

The main purpose of such an encryption as given in Eq. (3) is to provide secure message transmission against eavesdropper, more specifically filtering techniques (References 23-24) as explained in Sec. V. It should be also noted that the encrypter described by Eq. (1) can be defined as an $n^{\text {th }}$ order system, in general. It is chosen as a third order system for consistency with the Lorenz system used for illustrations in Sec. IV.

\section{II.B. Receiver Section}

An observer as defined in Eq. (4) below is used to rebuild the chaotic decrypter's state variables

$$
\dot{y}=A y+g(y)+\sigma(e),
$$

where

$\boldsymbol{y} \in R^{3}=$ chaotic decrypter state vector such that $\|\boldsymbol{y}\|<d_{1}$ for some $d_{1}>0$,

$A \in R^{3 \times 3}=$ same as given for Eq. (1),

$\boldsymbol{e} \in R^{3}=$ state error vector,

$\boldsymbol{\sigma}(\boldsymbol{e}) \in R^{3}=$ error control law.

The state error $\boldsymbol{e} \in R^{3}$ is defined as

$$
e=y-x
$$

where $\boldsymbol{e}=\left[e_{1}, e_{2}, e_{3}\right]^{T}$ with $e_{1}=y_{1}-x_{1}, e_{2}=y_{2}-x_{2}$ and $e_{3}=y_{3}-x_{3}$. Since the state vectors are bounded with $d_{1}>0$, the state error vector is also bounded, namely, $\|\boldsymbol{e}\|=$ 
$\|\boldsymbol{y}-\boldsymbol{x}\|<2 d_{1}$ for some $d_{1}>0$. It should be noted that since $x_{1}$ is directly received by the receiver as it is chosen to be the synchronization signal, only $e_{1}$ is known by the controller and $\boldsymbol{\sigma}(\boldsymbol{e})=\boldsymbol{\sigma}\left(e_{1}\right)$ for this case.

Here, the problem is to design the control law $\boldsymbol{\sigma}(\boldsymbol{e})$ in Eq. (4) so that the controlled chaotic decrypter state $\boldsymbol{y}$ tracks the chaotic encrypter state $\boldsymbol{x}$ by taking the error $\boldsymbol{e}$ to zero. For this aim, a control law $\boldsymbol{\sigma}(\boldsymbol{e})$ is proposed as

$$
\boldsymbol{\sigma}(\boldsymbol{e})=-K(C \boldsymbol{y}-z)
$$

where $K \in R^{3 x 1}$ is a constant gain matrix such that $\bar{A}=A-K C$ is stable, i.e. the eigenvalues $\lambda_{i}(i=1,2,3)$ of the matrix $\bar{A}$ are negative $\left(\lambda_{i}<0\right)$, via the choice of a proper gain matrix $K \in R^{3 x 1}$. Such a choice is possible if $(A, C)=\left[C, C A, C A^{2}\right]^{T}$ is observable.

Proposition 1: For system (1) and observer (4), the control law (6) provides a sufficient condition for the convergence of the error (5) to zero as time tends to infinity if $(A, C)$ is observable, and the Lipschitz condition (2) holds.

Proof: See Appendix A.

To obtain the message signal from the received signals, an inverse transformation of Eq. (3) is performed to obtain the transmitted message signal $\tilde{n}$, i.e.

$$
\tilde{n}=s^{-1}(s, \boldsymbol{y})=\frac{1}{\tau}\left(s-y_{2}-y_{3}\right)
$$

where 
$\tilde{n}=$ recovered message signal which is identical to the noisy message signal $n$ after synchronization is achieved, $s=$ encrypted message signal.

Here we also should note that the method can be generalized for $n$th order chaotic systems, and multiple synchronization signals.

\section{II.C. MOS and Its Reconstruction}

The MOS under consideration has the dynamical form

$$
\dot{\boldsymbol{r}}=M \boldsymbol{r}+\boldsymbol{m}(\boldsymbol{r}) \quad \text { and } \quad \boldsymbol{z}=C \boldsymbol{r}
$$

where

$\boldsymbol{r} \in R^{n}=$ state vector of the MOS such that $\|\boldsymbol{r}\|<d_{2}$ for some $d_{2}>0$,

$M \in R^{n x n}=$ constant system matrix,

$\boldsymbol{z} \in R^{m}=$ system output,

$C \in R^{m x n}=$ output matrix,

$\boldsymbol{m}: R^{n} \rightarrow R^{n}=$ nonlinear part of the MOS which satisfies the Lipschitz condition with Lipschitz constant $\mu_{2}>0$ for all $\boldsymbol{r}$ and $\hat{\boldsymbol{r}}$ such that $\|\boldsymbol{m}(\hat{\boldsymbol{r}})-\boldsymbol{m}(\boldsymbol{r})\| \leq \mu_{2}\|\hat{\boldsymbol{r}}-\boldsymbol{r}\|$.

In order to reconstruct the state variables of the MOS after decryption, the nonlinear observer given in Eqs. (4)-(6) will be used for the MOS model given in Eq. (8) as

$$
\dot{\hat{\boldsymbol{r}}}=M \hat{\boldsymbol{r}}+\boldsymbol{m}(\hat{\boldsymbol{r}})+\sigma(\boldsymbol{e}),
$$

where 
$\hat{\boldsymbol{r}}=$ estimate of $\boldsymbol{r}$ given in Eq. (8),

$\boldsymbol{e}=$ state error vector defined as

$$
\boldsymbol{e}=\hat{\boldsymbol{r}}-\boldsymbol{r},
$$

which is bounded such that $\|\boldsymbol{e}\|<2 d_{2}$ for some $d_{2}>0$ (since $\|\boldsymbol{r}\|<d_{2}$ ), and $\boldsymbol{\sigma}(\boldsymbol{e})$ is defined in a similar manner to Eq. (6) as

$$
\boldsymbol{\sigma}(\boldsymbol{e})=-\widehat{K}(C \hat{\boldsymbol{r}}-\boldsymbol{z})
$$

Again, $\widehat{K} \in R^{n x m}$ in Eq. (11) is chosen such that $\bar{M}=M-\bar{K} C$ is stable. Then Proposition 1 shows that the control law (11) provides a sufficient condition for the convergence of the error (10) to zero as time tends to infinity.

\section{THE EXAMPLE MOS}

The following reduced order BWR model (Ref. 33) is considered as an example MOS

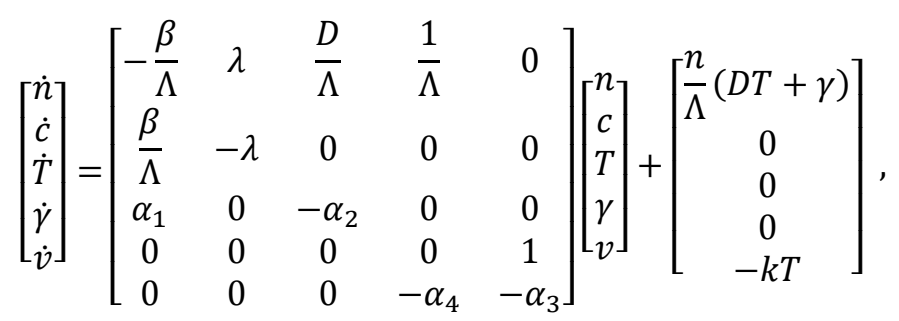

where $n$ is the only measurement to be sent to the receiver via chaotic encryption, and thus the output matrix in Eq. (6) is $C=\left[\begin{array}{lllll}1 & 0 & 0 & 0 & 0\end{array}\right]$. In Eq. (12),

$n=$ excess neutron population normalized to the steady state neutron density, $c=$ excess delayed neutron precursors concentration normalized to the steady state neutron density, 
$T=$ excess average fuel temperature,

$\gamma=$ coolant density

$v=$ excess void reactivity feedback.

The BWR system output $n$ is the message to be transmitted. At this point, it should be indicated that, for the purposes of this paper, Eq. (12) is used to represent both the MOS and its reconstructed surrogate at the receiver end. In actual applications, the model will only represent the reconstructed system. The nonlinear part of Eq. (12) can be represented with the function $\boldsymbol{m}(\boldsymbol{r})=\left[\frac{D}{\Lambda} n T+\frac{1}{\Lambda} n \gamma, 0,0,0,-k T\right]^{T}$. This function is continuously differentiable on $R^{5}$ and if the state variables satisfy $\|\boldsymbol{r}\|<d_{2}, \boldsymbol{m}(\boldsymbol{r})$ satisfies the Lipschitz condition as described in Sec.

II.C for a Lipschitz constant $\mu_{2}=\frac{2}{\Lambda} d_{2}$. We verify this in Appendix B. The example system parameters which were obtained from Vermont Yankee nuclear reactor (Ref. 33) are given in Table I. The parameter $k\left(K^{-1} S^{-2}\right)$ is a time-varying parameter and is related to the void reactivity coefficient and the fuel-to-coolant heat transfer coefficient. This parameter determines the stability of the reactor model. Since $k$ can change during the operation of the plant, it is assumed that $k$ is only approximately known.

Table I

Model parameters for Vermont Yankee Test $7 \mathrm{~N}^{*}$

\begin{tabular}{|l|l||l|l|}
\hline $\begin{array}{c}\text { Model } \\
\text { Parameters }\end{array}$ & \multicolumn{1}{|c||}{ Values } & $\begin{array}{c}\text { Model } \\
\text { Parameters }\end{array}$ & \multicolumn{1}{|c|}{ Values } \\
\hline \hline $\boldsymbol{\alpha}_{\mathbf{1}}\left(\boldsymbol{K \boldsymbol { s } ^ { - \mathbf { 1 } } )}\right.$ & 25.04 & $\boldsymbol{D}\left(\boldsymbol{K}^{-\mathbf{1}}\right)$ & $-2.52 \times 10^{-5}$ \\
\hline $\boldsymbol{\alpha}_{\mathbf{2}}\left(\boldsymbol{s}^{\mathbf{- 1}}\right)$ & 0.23 & $\boldsymbol{\beta}$ & 0.0056 \\
\hline $\boldsymbol{\alpha}_{\mathbf{3}}\left(\boldsymbol{s}^{\mathbf{- 1}}\right)$ & 2.25 & $\boldsymbol{\Lambda}\left(\boldsymbol{s}^{\mathbf{- 1}}\right)$ & $4 \times 10^{-5}$ \\
\hline $\boldsymbol{\alpha}_{\mathbf{4}}\left(\boldsymbol{s}^{\mathbf{- 2}}\right)$ & 6.82 & $\boldsymbol{\lambda}\left(\boldsymbol{s}^{\mathbf{1}}\right)$ & 0.08 \\
\hline
\end{tabular}

*Ref. 33

The BWRs can exhibit instabilities at low flow and high power conditions. ${ }^{34-36}$ The magnitude of the feedback can cause oscillations to diverge exponentially and can make the reactor unstable. The rich dynamics of the BWR system and its sensitivity to $k$ are depicted in Fig. 2 
for the initial conditions $\boldsymbol{r}(0)=[4,150,30,0,0.04]^{T}$. Fig. 2a shows a stable behavior (stable focus) for $k=3 \times 10^{-3}$. Fig. 2b shows a limit cycle (period-1) behavior for $k=4.2 \times 10^{-3}$. Fig. 2c shows a period-2 motion for $k=6 \times 10^{-3}$. Fig. $2 \mathrm{~d}$ shows a chaotic behavior for $k=$ $7.2 \times 10^{-3}$

Figure 2

\section{AN APPLICATION WITH NUMERICAL SIMULATIONS}

For the transmitter section, the Lorenz system (Ref. 37) is used as a chaotic encrypter, i.e.

$$
\left[\begin{array}{l}
\dot{x}_{1} \\
\dot{x}_{2} \\
\dot{x}_{3}
\end{array}\right]=\left[\begin{array}{ccc}
-a & a & 0 \\
b & -1 & 0 \\
0 & 0 & -c
\end{array}\right]\left[\begin{array}{l}
x_{1} \\
x_{2} \\
x_{3}
\end{array}\right]+\left[\begin{array}{c}
0 \\
-x_{1} x_{3} \\
x_{1} x_{2}
\end{array}\right]
$$

where the system parameters $a, b, c$ are designed as nonlinear functions of the state variables as

$$
a=0.1 x_{2}+10, \quad b=0.1 x_{3}+25, \quad c=0.04 x_{1}+1.1
$$

In Eq. (13), the first state variable $x_{1}$ is selected as synchronization signal, which is completely independent of the third state variable $x_{3}$. Substituting (14) into Eq. (13), a new chaotic system (a modified form of the Lorenz system) can be obtained as

$$
\left[\begin{array}{l}
\dot{x}_{1} \\
\dot{x}_{2} \\
\dot{x}_{3}
\end{array}\right]=\left[\begin{array}{ccc}
-10 & 10 & 1 \\
25 & -1 & 0 \\
0 & 0 & -1.1
\end{array}\right]\left[\begin{array}{l}
x_{1} \\
x_{2} \\
x_{3}
\end{array}\right]+\left[\begin{array}{c}
0.1 x_{2}\left(x_{2}-x_{1}\right)-x_{3} \\
-0.9 x_{1} x_{3} \\
x_{1} x_{2}-0.04 x_{1} x_{3}
\end{array}\right]
$$

The nonlinear part of Eq. (15) can be represented with the function $\boldsymbol{g}(\boldsymbol{x})=\left[0.1 x_{2}\left(x_{2}-x_{1}\right)-\right.$ $\left.x_{3},-0.9 x_{1} x_{3}, x_{1} x_{2}-0.04 x_{1} x_{3}\right]^{T}$. This function is continuously differentiable on $R^{3}$ and if the state variables satisfy $\|\boldsymbol{x}\|<d_{1}, \boldsymbol{g}(\boldsymbol{x})$ satisfies the Lipschitz condition as described in Eq. (2) for a Lipschitz constant $\mu_{1}=2 d_{1}$, as shown in Appendix C. When the parameters are 
selected as nonlinear functions of the state variables, a care must be taken to retain the chaotic nature of the system. The behavior of the new chaotic system given by Eq. (15) is illustrated in Fig. 3 which shows that the new system given in Eq. (15) still retains the typical double-scroll attractor of the Lorenz system. The time variations of the key parameters in Eq. (14) are shown in Fig. 4. It is seen that all three parameters have chaotic variations as a function of time, which makes the chaotic communication securer against eavesdroppers (see Sec. V).

\section{Figure 3}

\section{Figure 4}

For decoding the signal $s$ that will be received at the receiver section (see Fig. 1) together with $x_{1}$, we can design the chaotic decrypter as

$$
\left[\begin{array}{l}
\dot{y}_{1} \\
\dot{y}_{2} \\
\dot{y}_{3}
\end{array}\right]=\left[\begin{array}{ccc}
-10 & 10 & 1 \\
25 & -1 & 0 \\
0 & 0 & -1.1
\end{array}\right]\left[\begin{array}{l}
y_{1} \\
y_{2} \\
y_{3}
\end{array}\right]+\left[\begin{array}{c}
0.1 y_{2}\left(y_{2}-y_{1}\right)-y_{3} \\
-0.9 y_{1} y_{3} \\
y_{1} y_{2}-0.04 y_{1} y_{3}
\end{array}\right]+\boldsymbol{\sigma}(\boldsymbol{e}),
$$

where $\boldsymbol{\sigma}(\boldsymbol{e}) \in R^{3}$ is given in Eq. (6).

To reconstruct the BWR state variables from the recovered message signal for tracking, we can use the following nonlinear observer from Eqs. (9)-(12),

$$
\left[\begin{array}{c}
\dot{\hat{n}} \\
\dot{\hat{c}} \\
\dot{\hat{T}} \\
\dot{\hat{\gamma}} \\
\dot{\hat{v}}
\end{array}\right]=\left[\begin{array}{ccccc}
-\frac{\beta}{\Lambda} & \lambda & \frac{D}{\Lambda} & \frac{1}{\Lambda} & 0 \\
\frac{\beta}{\bar{\Lambda}} & -\lambda & 0 & 0 & 0 \\
\alpha_{1} & 0 & -\alpha_{2} & 0 & 0 \\
0 & 0 & 0 & 0 & 1 \\
0 & 0 & 0 & -\alpha_{4} & -\alpha_{3}
\end{array}\right]\left[\begin{array}{c}
\hat{n} \\
\hat{c} \\
\hat{T} \\
\hat{\gamma} \\
\hat{v}
\end{array}\right]+\left[\begin{array}{c}
\hat{n}(D \hat{T}+\hat{\gamma}) \\
0 \\
0 \\
0 \\
-\hat{k} \widehat{T}
\end{array}\right]+\boldsymbol{\sigma}(\hat{\boldsymbol{e}})
$$

where

$(\hat{n}, \hat{c}, \hat{T}, \hat{\gamma}, \hat{v})=$ estimates of the $(n, c, T, \gamma, v)$, 
$\hat{k}=$ estimated value of the unknown system parameter $k$.

Numerical simulations are performed by using SIMULINK. The values of the gain matrices $K$ and $\widehat{K}$ are given in Table II. In numerical simulations, the noise on the outgoing message signal is assumed to be normally distributed with a mean of 0 and a standard deviation of 1 , which leads to fluctuations in the encrypted message signal. Here the difficulty is to select the value of the observer parameter $\hat{k}$ when the actual parameter of the BWR system $k$ is not known. Fortunately, we can select this parameter as a constant due to robust nature of the nonlinear observer, say $\hat{k}=6.2 \times 10^{-3}\left(K^{-1} S^{-2}\right)$. We will see that the estimation error will be very small when the reactor is operated with $k$ within a fairly large interval around $\hat{k}$ (i.e. $4.2 \times 10^{-3} \leq$ $\left.\hat{k} \leq .7 .2 \times 10^{-3}\right)$. Numerical simulations are realized for three different values of $k$.

\section{Table II}

Gain matrices $K$ and $\widehat{K}$ (see Eq. (6) and (11))

\begin{tabular}{|c|c|}
\hline Gain Matrix & Values \\
\hline$K$ & {$[400,0,0]^{T}$} \\
\hline$\widehat{K}$ & {$[500,0,0,0,0]^{T}$} \\
\hline
\end{tabular}

Case 1: The parameter $k$ is selected as $k=4.2 \times 10^{-3}\left(K^{-1} S^{-2}\right)$ for a periodic BWR system behavior. Numerical results are presented in Figs. 5 and 6. Fig. 5 shows the response of the proposed communication method. Fig. 5a displays the encrypted message signal with noise. Fig. $5 b$ displays the original message signal without noise and Fig. $5 \mathrm{c}$ displays the decrypted message signal with noise removed using a low pass filter. Comparison of the original and decrypted message signals shows that the match between the transmitted and recovered messages is very good. Fig. 6 shows the time responses of the state variables of the BWR system and its estimator (i.e. Eq. (17)). Fig. 6a indicates good match between $n$ and $\hat{n}$ (and for other state variables) starting from about 5 seconds. Also, note that since the reactor system is 
highly nonlinear and the behavior is limit cycle, the small observer error causes higher amplitudes on observer state variables $\hat{\gamma}$ and $\hat{v}$, but still the observer follows the frequency of the variations correctly and the differences have almost no effect on the estimated output of interest $(n)$ even though there is a factor about 1.5 difference between $k=4.2 \times 10^{-3}$ of the actual system and $\hat{k}=6.2 \times 10^{-3}$ of the observer.

Figure 5

Figure 6

Case 2: The system parameter $k$ is selected as $k=6 \times 10^{-3}\left(K^{-1} S^{-2}\right)$ for a period-2 limit cycle behavior. Numerical results are presented in Figs. 7 and 8. Comparison of Figs. 7b and 7c shows that the transmitted and recovered message signals match well. Fig. 8 shows that the reconstructed and actual system behaviors are identical after $t=5$ seconds. It is interesting to note that while there is a small difference between the actual $k=6 \times 10^{-3}$ and assumed $\hat{k}=6.2 \times 10^{-3}, k$ corresponds to a period-2 limit cycle and $\hat{k}$ is a period- 8 limit cycle.

\section{Figure 7}

\section{Figure 8}

Case 3: The system parameter $k$ is selected as $k=7.2 \times 10^{-3}\left(K^{-1} S^{-2}\right)$ for a chaotic BWR system behavior (note that its observer counterpart $\hat{k}=6.2 \times 10^{-3}$ still corresponds to a limit cycle behavior). Numerical results are presented in Figs. 9 and 10. While the system parameter $k$ is $16 \%$ larger than $\hat{k}$ and Fig. 2 shows how sensitive the system behavior is to such changes, Fig. 10 shows that differences between the original and reconstructed system behaviors are still very small which demonstrate that the observer is robust in the presence of both parameter uncertainty and observation noise. 


\section{Figure 9}

\section{Figure 10}

\section{THE SECURITY OF THE SCHEME AGAINST PASSIVE EAVESDROPPING: TWO CASE STUDIES}

In the literature, many papers are available which address the cryptanalysis of the chaos-based communication methods. ${ }^{22-32}$ The main possibilities for cryptanalysis are extracting message signal directly from the transmitted ciphertext signal, extracting the chaotic carrier signal to recover the message signal by removing the carrier signal from the transmitted ciphertext signal, and estimating the key (or secret) parameters from the transmitted signals to break the cryptosystem. $^{32}$ Low pass filters, high pass filters and generalized (or other known) synchronization methods have been proposed to break chaos-based secure communication methods. In this section, two tests will be performed to analyze the security of the proposed method under the assumptions that a cryptanalyst obtains both synchronization and encrypted message signals, and has the knowledge about type of the chaotic encrypter used in the communication. Firstly, a procedure outlined in Ref. 24 is followed to show that using filtering techniques is not possible to break the method proposed in this paper. The Butterworth low pass filter (Ref. 38) with different orders and cutoff frequencies are applied to the encrypted message of Case 1 (Fig. 11a). The result for a 4th order Butterworth low pass filtering with cutoff frequency of $6 \mathrm{rad} / \mathrm{sec}$ (due to the frequency of the message signal in Case 1) is given in Fig. 11b. The normalized mean square error (NMSE) is $\frac{\int\left(n-s_{L P F}\right)^{2} d t}{\int n^{2} d t}=86.43$, where $n$ is the message signal, and $s_{L P F}$ is the low pass filtered signal (for a minimum NMSE, the filtered signal $s_{L P F}$ is subtracted from its average value), which shows that the energy of the error is 
intolerably high compared to the energy of the message signal. From the ratio of the NMSE and the comparison of Figs. $11 \mathrm{~b}$ and $11 \mathrm{~d}$, we can say that the filtered signal does not exhibit any similarities with the original message signal.

In our second case study, we assume that the eavesdropper managed to obtain perfect knowledge of the linear parameters of the encrypter. We illustrate that, even in such a case, the eavesdropper cannot extract a reasonably close replica of the original signal. Namely, we assume that the eavesdropper tries the same synchronization method in the form of Eq. (18), which includes the constant parts of the variable parameters as given in Eq. (14), and the same inverse transformation of Eq. (7) $\left(s^{-1}\right.$ in Fig. 1) to extract information, i.e.

$$
\left[\begin{array}{l}
\dot{y}_{1} \\
\dot{y}_{2} \\
\dot{y}_{3}
\end{array}\right]=\left[\begin{array}{ccc}
-10 & 10 & 0 \\
25 & -1 & 0 \\
0 & 0 & -1.1
\end{array}\right]\left[\begin{array}{l}
y_{1} \\
y_{2} \\
y_{3}
\end{array}\right]+\left[\begin{array}{c}
0 \\
-y_{1} y_{3} \\
y_{1} y_{2}
\end{array}\right]+\boldsymbol{\sigma}(\boldsymbol{e})
$$

The NMSE for this case is $\frac{\int\left(n-\tilde{n}_{i n t}\right)^{2} d t}{\int n^{2} d t}=26.5$, where $n$ is the message signal and $\tilde{n}_{\text {int }}$ is the extracted signal by the intruder, which shows that the MSE is an order of magnitude higher than the energy of the original system, i.e. the eavesdropper fails to obtain a reasonably close replica of the original signal. Comparison of Figs. 11c and 11d illustrate this observation graphically.

\section{Figure 11}

\section{CONCLUSION}

A chaos-based secure communication method and a long-distance monitoring scheme for the use in nuclear engineering applications are proposed to facilitate research, evaluation, data 
storage, fault detection and other related usages. Due to their high parameter and initial condition sensitivity, and significant unpredictability, chaotic systems have been widely utilized for encryption in communication systems, however are susceptible to noisy data. In this paper we develop a robust chaotic encryption system for long-distance monitoring of nuclear reactors in the presence of noisy data. The proposed chaotic transmitter output states are nonlinear functions of the system parameters. The basic security analyses show that the proposed system is secure with respect to a number of common passive eavesdropping strategies. In this study, we present and illustrate a third order chaotic system as encrypter and decrypter, however, the method can be generalized to $n$th order chaotic systems. 


\section{REFERENCES}

1. B. P. HALLBERT, J. J. PERSENSKY, C. SMIDTS, T. ALDEMIR and J. NASER, Report from the Light Water Reactor Sustainability Workshop on Advanced Instrumentation, Information, and Control Systems and Human-System Interface Technologies, INL/EXT-0916631, Idaho National Laboratory, Idaho, (2009).

2. S. M. MODRO, J. E. FISHER, K. D. WEAVER, J. N. REYES, J. T. GROOME, P. BABKA and T. M. CARLSON, "Multi-Application Small Light Water Reactor Final Report," INEEL/EXT-04-01626, (2003).

3. J. C. KUIJPER, "PUMA - Plutonium and Minor Actinides Management in Thermal High-Temperature Reactors,” ENC2007, European Nuclear Society, Brussels, Belgium, (2007).

4. H. TAKAHASHI, "Toward the 21st century nuclear-science technology," Progress in Nuclear Energy, 47, 1-4, 53 (2005).

5. M. J. OGORZALEK, Chaos and Complexity in Nonlinear Electronic Circuits, World Scientific (1997).

6. P. STAVROULAKIS, Chaos Applications in Telecommunications, Taylor\&Francis (2006).

7. E. M. ElABBASY, H. N. AGIZA and M. M. EL-DESSOKY, "Global chaos synchronization for four scroll attractor by nonlinear control," Sci. Res. Essay, 1, 3, 65 (2006). 
8. A. M. HARB and W. M. AHMAD, "Chaotic systems synchronization in secure communication systems," Proc. World Congress in Computer Science Computer Engineering, and Applied Computing, Las Vegas, (2006).

9. D. GHOSH, S. BANERJEE and A. R. CHOWDHURY, "Synchronization between variable time-delayed systems and cryptography," Euro Phys. Lett., 80, 3, in CD (2007).

10. G. ABLAY and T. ALDEMIR, "Synchronization of different chaotic systems using generalized active control," IEEE Conference on Electrical, Electronics Engineering, ELECO'09, vol. II, 182 (2009).

11. W. PERRUQUETTI, T. FLOQUET and E. MOULAY, "Finite-Time Observers: Application to Secure Communication," IEEE Trans. Automatic Control, 53 , 1, 356 (2008).

12. M. RAFIKOV and J. M. BALTHAZAR, "On control and synchronization in chaotic and hyperchaotic systems via linear feedback control," Commun. Nonlinear Sci. Numer. Simulat., 13, 1246 (2008).

13. L. MIN and J. JING, "A new theorem to synchronization of unified chaotic systems via adaptive control," Chaos, Solitons Fractals, 24, 5, 1363(2004).

14. K. CUOMO and A. OPPENHEIM, "Circuit implementation of synchronized chaos with applications to communications," Phys. Rev. Lett., 71, 65 (1993).

15. Q. MEMON, "Synchronized chaos for network security," Computer Communications, 26, 498 (2003).

16. E. ALVAREZ, A. FERNÁNDEZ, P. GARCÍA, J. JIMÉNEZ and A. MARCANO, "New approach to chaotic encryption," Phys. Lett. A, 263, 373 (1999). 
17. Z. H. GUAN, F. HUANG and W. GUAN, "Chaos-based image encryption algorithm," Phys. Lett. A, 346, 153 (2005).

18. M. FEKI, "An adaptive chaos synchronization scheme applied to secure communication," Chaos, Solitons and Fractals, 18, 1, 141 (2003).

19. M. I. SOBHY and A. R. SHEHATA, "Chaotic algorithm for data encryption," IEEE International Conference on Acoustics, Speech, and Signal Processing, vol. 1, p. i-xcii, (2001).

20. J. M. GRZYBOWSKI, M. RAFIKOV and J. M. BALTHAZAR, "Synchronization of the unified chaotic system and application in secure communication," Commun Nonlinear Sci Numer Simulat, 14, 2793 (2009).

21. A. A. ZAHER, "An improved chaos-based secure communication technique using a novel encryption function with an embedded cipher key," Chaos, Solitons and Fractals, 42, 2804 (2009).

22. C. ÇOKAL and E. SOLAK, "Cryptanalysis of a chaos-based image encryption algorithm," Physics Letters A, 373, 1357 (2009).

23. G. ÁlvareZ, S. LI, F. MONTOYA, G. PASTOR and M. ROMERA, "Breaking projective chaos synchronization secure communication using filtering and generalized synchronization," Chaos, Solitons and Fractals, 24, 3, 775 (2005).

24. G. ÁlvAREZ, F. MONTOYA, M. ROMERA and G. PASTOR, "Breaking parameter modulated chaotic secure communication system," Chaos, Solitons and Fractals, 21, 4, 783 (2004). 
25. S. LI, G. ÁLVAREZ and G. CHEN, "Breaking a chaos-based secure communication scheme designed by an improved modulation method," Chaos, Solitons and Fractals, 25, 1, 109 (2005).

26. C. LI, S. LI, G. ALVAREZ, G. CHEN and K. LO, "Cryptanalysis of a chaotic block cipher with external key and its improved version," Chaos, Solitons and Fractals, 37, 1, 299 (2008).

27. M. LEI, G. MENG and Z. FENG, "Security analysis of chaotic communication systems based on Volterra-Wiener-Korenberg model," Chaos, Solitons and Fractals, 28, 1, 264 (2006).

28. G. ÁlVAREZ, F. MONTOYA, M. ROMERA and G. PASTOR, “Cryptanalyzing an improved security modulated chaotic encryption scheme using ciphertext absolute value," Chaos, Solitons and Fractals, 23, 5, 1749 (2005).

29. F. HUANG and Z. GUAN, "Cryptosystem using chaotic keys," Chaos, Solitons and Fractals, 23, 3, 851 (2005).

30. A. ALI PACHA, N. HADJ-SAID, B. BELMEKI and A. BELGORAF, "Chaotic behavior for the secret key of cryptographic system," Chaos, Solitons and Fractals, 23, 5, 1549 (2005).

31. M. I. SOBHY and A. R. SHEHATA, "Methods of attacking chaotic encryption and countermeasures," IEEE International Conference on Acoustics, Speech, and Signal Processing, vol.2, p.1001, (2001).

32. G. ALVAREZ and S. LI, "Some Basic Cryptographic Requirements for Chaos-Based Cryptosystems," Int J Bifurc Chaos Appl Sci Eng, 16, 8, 2129 (2006). 
33. J. MARCH-LEUBA, "Nonlinear dynamics and chaos in boiling water reactors," NATO Advanced Research Workshop on Noise and Nonlinear Phenomena in Nuclear Systems, Spain, p.371, (1988).

34. C. DEMAZIERE, C. MARCEL, M. ROHDE and T. VAN DER HAGEN, "Multifractal analysis of chaotic flashing-induced instabilities in boiling channels in the natural-circulation CIRCUS facility," Nuclear Science and Engineering, 158, 164 (2008).

35. G. V. PRASAD and M. PANDEY, "Stability analysis and nonlinear dynamics of natural circulation boiling water reactors," Nuclear Engineering and Design, 238, 229 (2008).

36. N. S. POSTNIKOV, "Possibility, admissability, and desirability of implementing pulsed stochastic regimes in boiling-water reactors with natural coolant circulation," Atomic Energy, 97, 6, 820 (2004).

37. E. N. LORENZ, "Deterministic nonperiodic flow," J. Atmospheric Science, 20, 130 (1963).

38. D. SCHLICHTHÄRLE, Digital Filters: Basics and Design, Springer (2000).

39. H. K. KHALIL, Nonlinear Systems, Third Ed., Prentice-Hall (2002).

40. B. A. FRANCIS, A Course in $H_{\infty}$ Control Theory, Lecture Notes in Control and Information Sciences, vol. 88, Springer-Verlag (1987). 


\section{APPENDIX A}

\section{PROOF OF PROPOSITION 1}

The error dynamic of Eq. (5) is

$$
\begin{aligned}
\dot{\boldsymbol{e}} & =\dot{\boldsymbol{y}}-\dot{\boldsymbol{x}} \\
& =A \boldsymbol{y}+\boldsymbol{g}(\boldsymbol{y})+\boldsymbol{\sigma}(\boldsymbol{e})-A \boldsymbol{x}-\boldsymbol{g}(\boldsymbol{x}) \\
& =A \boldsymbol{e}+(\boldsymbol{g}(\boldsymbol{y})-\boldsymbol{g}(\boldsymbol{x}))-K(C \boldsymbol{y}-z) \\
& =\bar{A} \boldsymbol{e}+(\boldsymbol{g}(\boldsymbol{y})-\boldsymbol{g}(\boldsymbol{x}))
\end{aligned}
$$

where $\bar{A}=A-K C$. For stability analysis of Eq. (A1), a candidate Lyapunov function (Ref. 39) $V(\boldsymbol{e})$ can be defined as

$$
V(\boldsymbol{e})=\boldsymbol{e}^{T} P \boldsymbol{e},
$$

where $P=P^{T}>0$ satisfies the Lyapunov equation for the Lyapunov pair $(P, Q)$, i.e.

$$
\bar{A}^{T} P+P \bar{A}+Q=0 .
$$

The derivative of Eq. (A2) must be negative for asymptotic convergence to $\boldsymbol{e}=0$ (Ref. 39, Theorem 4.1). Then

$$
\begin{aligned}
\dot{V}(\boldsymbol{e}) & =\boldsymbol{e}^{T} P \dot{\boldsymbol{e}}+\dot{\boldsymbol{e}}^{T} P \boldsymbol{e} \\
& =\boldsymbol{e}^{T} P(\bar{A} \boldsymbol{e}+(\boldsymbol{g}(\boldsymbol{y})-\boldsymbol{g}(\boldsymbol{x})))+(\bar{A} \boldsymbol{e}+(\boldsymbol{g}(\boldsymbol{x})-\boldsymbol{g}(\boldsymbol{y})))^{T} P \boldsymbol{e} \\
& =\boldsymbol{e}^{T}\left(P \bar{A}+\bar{A}^{T} P\right) \boldsymbol{e}+\boldsymbol{e}^{T} P(\boldsymbol{g}(\boldsymbol{x})-\boldsymbol{g}(\boldsymbol{y}))+(\boldsymbol{g}(\boldsymbol{x})-\boldsymbol{g}(\boldsymbol{y}))^{T} P \boldsymbol{e} .
\end{aligned}
$$

For any two vectors $\boldsymbol{N}$ and $\boldsymbol{M}$ of the same dimension and any $\epsilon>0$, one can obtain

$$
\begin{gathered}
\epsilon\left(\frac{1}{\epsilon} \boldsymbol{M}-\boldsymbol{N}\right)^{T}\left(\frac{1}{\epsilon} \boldsymbol{M}-\boldsymbol{N}\right) \geq 0 \\
\Rightarrow \boldsymbol{M}^{T} \boldsymbol{N}+\boldsymbol{N}^{T} \boldsymbol{M} \leq \frac{1}{\epsilon} \boldsymbol{M}^{T} \boldsymbol{M}+\epsilon \boldsymbol{N}^{T} \boldsymbol{N} .
\end{gathered}
$$

In addition, the following properties will be used in the sequel:

$$
\begin{gathered}
\boldsymbol{e}^{T} \boldsymbol{e}=\|\boldsymbol{e}\|^{2}, \\
\|M N\| \leq\|M\|\|N\| \quad \text { (Cauchy's inequality) }
\end{gathered}
$$

Using Eq. (A5) (i.e. taking $\boldsymbol{N}=P \boldsymbol{e}, \boldsymbol{M}=\boldsymbol{g}(\boldsymbol{y})-\boldsymbol{g}(\boldsymbol{x})$ ), Eq. (A6), and the Lipschitz condition (2), one can obtain that

$$
\dot{V}(\boldsymbol{e}) \leq \boldsymbol{e}^{T}\left(\bar{A}^{T} P+P \bar{A}\right) \boldsymbol{e}+\epsilon(P \boldsymbol{e})^{T} P \boldsymbol{e}+\frac{1}{\epsilon}(\boldsymbol{g}(\boldsymbol{y})-\boldsymbol{g}(\boldsymbol{x}))^{T}(\boldsymbol{g}(\boldsymbol{y})-\boldsymbol{g}(\boldsymbol{x}))
$$




$$
\begin{aligned}
& =\boldsymbol{e}^{T}\left(\bar{A}^{T} P+P \bar{A}\right) \boldsymbol{e}+\epsilon \boldsymbol{e}^{T} P^{2} \boldsymbol{e}+\frac{1}{\epsilon}\|\boldsymbol{g}(\boldsymbol{y})-\boldsymbol{g}(\boldsymbol{x})\|^{2} \\
& \leq \boldsymbol{e}^{T}\left(\bar{A}^{T} P+P \bar{A}\right) \boldsymbol{e}+\epsilon \boldsymbol{e}^{T} P^{2} \boldsymbol{e}+\frac{1}{\epsilon} \mu_{1}^{2}\|\boldsymbol{e}\|^{2} \\
& =\boldsymbol{e}^{T}\left(\bar{A}^{T} P+P \bar{A}+P(\epsilon I) P+\frac{\mu_{1}^{2}}{\epsilon} I\right) \boldsymbol{e}
\end{aligned}
$$

where $I \in R^{3 \times 3}$ is the identity matrix. Since $\bar{A}$ is stable and $(\bar{A}, \epsilon I)$ is controllable (i.e. $\operatorname{rank}\left(\epsilon I, \epsilon \bar{A} I, \epsilon \bar{A}^{2} I\right)=3$ ), there exists a positive definite symmetric solution $P$ to the algebraic Riccati equation (Ref. 40, Chapter 7, Theorem 2 and Corollary 1)

$$
\bar{A}^{T} P+P \bar{A}+P(\epsilon I) P+\frac{\mu_{1}^{2}}{\epsilon} I=0 .
$$

which indicates an entry-wise equality to zero, and hence $\dot{V}(\boldsymbol{e}) \leq 0$. Therefore, the error $\boldsymbol{e}$ asymptotically converges zero as time tends to infinity.

\section{APPENDIX B}

\section{A LIPSCHITZ CONSTANT FOR THE MOS SYSTEM}

The nonlinear part of Eq. (12) is continuously differentiable on $R^{5}$ since

$$
\boldsymbol{m}(\boldsymbol{r})=\left[\begin{array}{c}
\frac{n}{\Lambda}(D T+\gamma) \\
0 \\
0 \\
0 \\
-k T
\end{array}\right] \Rightarrow\left[\frac{\partial \boldsymbol{m}}{\partial \boldsymbol{r}}\right]=\left[\begin{array}{ccccc}
\frac{D}{\Lambda} T+\frac{1}{\Lambda} \gamma & 0 & \frac{D}{\Lambda} n & \frac{1}{\Lambda} n & 0 \\
0 & 0 & 0 & 0 & 0 \\
0 & 0 & 0 & 0 & 0 \\
0 & 0 & 0 & 0 & 0 \\
0 & 0 & -k & 0 & 0
\end{array}\right]
$$

A Lipschitz constant can be calculated using knowledge of $[\partial \boldsymbol{m} / \partial \boldsymbol{r}]$ (Ref. 39), and using the fact that all state variables are less than $d_{2}$ since we assume that $\|\boldsymbol{r}\|<d_{2}$. From $\|\cdot\|_{\infty}$ (infinity norm) for vectors in $R^{5}$ and the induced matrix norm for matrices, we have

$$
\left\|\frac{\partial \boldsymbol{m}}{\partial \boldsymbol{r}}\right\|_{\infty}=\max \left\{\left|\frac{D}{\Lambda} T+\frac{1}{\Lambda} \gamma\right|+\left|\frac{D}{\Lambda} n\right|+\left|\frac{1}{\Lambda} n\right|,|-k|\right\} \leq \frac{2}{\Lambda} d_{2}
$$


where $D \ll 1$ (see Table I). Therefore, a Lipschitz constant can be taken as $\mu_{2}=\frac{2}{\Lambda} d_{2}$. Note that the choice of a norm on $R^{n}$ does not affect the Lipschitz property of a function due to the equivalence of norms, but it only affects the value of Lipschitz constant (Ref. 39).

\section{APPENDIX C}

\section{A LIPSCHITZ CONSTANT FOR THE CHAOTIC DECRYPTER}

The nonlinear part of Eq. (15) is continuously differentiable on $R^{3}$ since

$$
\boldsymbol{g}(\boldsymbol{x})=\left[\begin{array}{c}
0.1 x_{2}\left(x_{2}-x_{1}\right)-x_{3} \\
-0.9 x_{1} x_{3} \\
x_{1} x_{2}-0.04 x_{1} x_{3}
\end{array}\right] \Rightarrow\left[\frac{\partial \boldsymbol{g}}{\partial \boldsymbol{x}}\right]=\left[\begin{array}{ccc}
-0.1 x_{1} & 0.2 x_{2}-0.1 x_{1} & -1 \\
-0.9 x_{3} & 0 & -0.9 x_{1} \\
x_{2}-0.04 x_{3} & x_{1} & -0.04 x_{1}
\end{array}\right]
$$

Similar to Appendix B, a Lipschitz constant can be found as $\mu_{1}=2 d_{1}$. 


\section{Caption of Figures}

Fig. 1. The proposed chaotic communication and long-distance monitoring scheme.

Fig. 2. The phase portraits of the BWR system in (n-T) space. (a) Stable focus for $k=$ $3 \times 10^{-3}\left(K^{-1} s^{-2}\right)$, (b) limit cycle for $k=4.2 \times 10^{-3}\left(K^{-1} s^{-2}\right)$, (c) period-2 motion for $k=6 \times 10^{-3}\left(K^{-1} s^{-2}\right),(\mathrm{d})$ chaotic motion for $k=7.2 \times 10^{-3}\left(K^{-1} s^{-2}\right)$.

Fig. 3. Dynamical behavior of the chaotic system of Eq. (15), (a) a double-scroll attractor of the chaotic system in the $\left(x_{1}-x_{3}\right)$ plane, (b) the time responses of state variables.

Fig. 4. The time behaviors of the key parameters $a=0.1 x_{2}+10, b=0.1 x_{3}+25, c=$ $0.04 x_{1}+1.1$, (a) the parameter $a,(\mathrm{~b})$ the parameter $b$, (c) the parameter $c$.

Fig. 5. The response of the proposed communication method for Case 1: (a) encrypted message signal with noise, (b) original message signal without noise and (c) decrypted message signal with noise removed using a low pass filter.

Fig. 6. The time responses of the BWR states and reconstructed states for Case 1: (a) $n$ and $\hat{n}$, (b) $c$ and $\hat{c}$, (c) $T$ and $\hat{T}$, (d) $\gamma$ and $\hat{\gamma}$, (e) $v$ and $\hat{v}$.

Fig. 7. The response of the proposed communication method for Case 2: (a) encrypted message signal with noise, (b) original message signal without noise and (c) decrypted message signal with noise removed using a low pass filter.

Fig. 8. The time responses of the BWR states and reconstructed states for Case 2: (a) $n$ and $\hat{n}$, (b) $c$ and $\hat{c}$, (c) $T$ and $\hat{T}$, (d) $\gamma$ and $\hat{\gamma}$, (e) $v$ and $\hat{v}$. 
Fig. 9. The response of the proposed communication method for Case 3: (a) encrypted message signal with noise, (b) original message signal without noise and (c) decrypted message signal with noise removed using a low pass filter.

Fig. 10. The time responses of the BWR states and reconstructed states for Case 3: (a) $n$ and $\hat{n}$, (b) $c$ and $\hat{c}$, (c) $T$ and $\widehat{T}$, (d) $\gamma$ and $\hat{\gamma}$, (e) $v$ and $\hat{v}$.

Fig. 11. The responses of the intruder systems for Case 1: (a) the encrypted message signal $s(t)$, (b) the output of the low-pass filter $s_{L P F}(t),(\mathrm{c})$ the output of the fixed parameter chaotic decrypter model of Eq. (18), $\tilde{n}_{\text {int }}(t)$, and (d) the original message signal $n(t)$. 
Figure 1

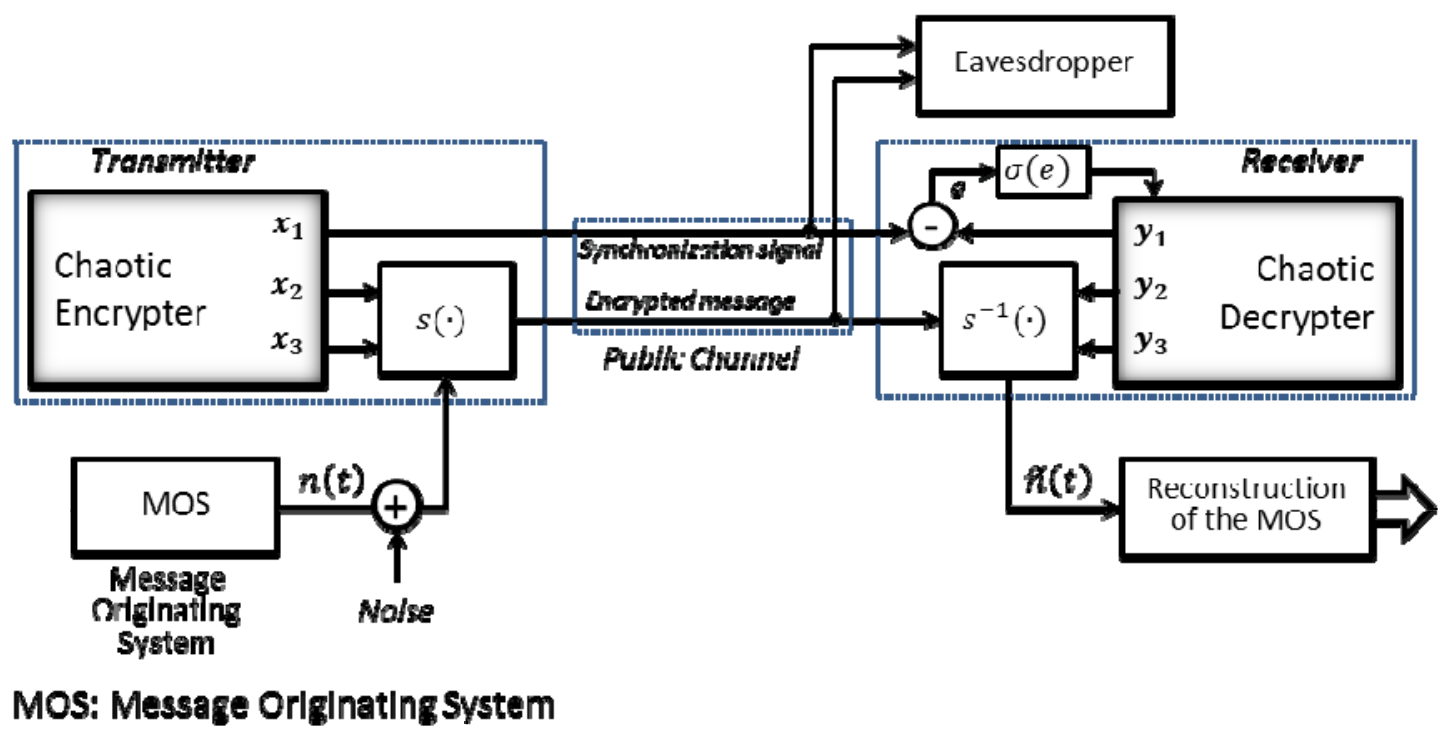


Figure 2

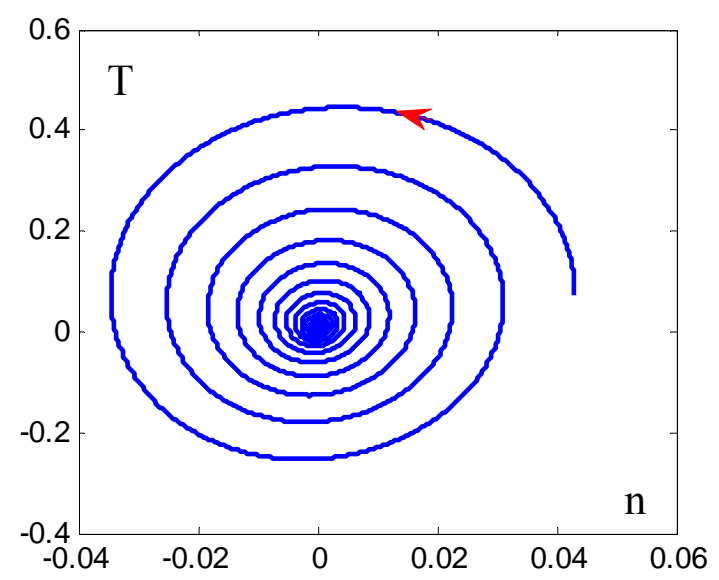

(a)

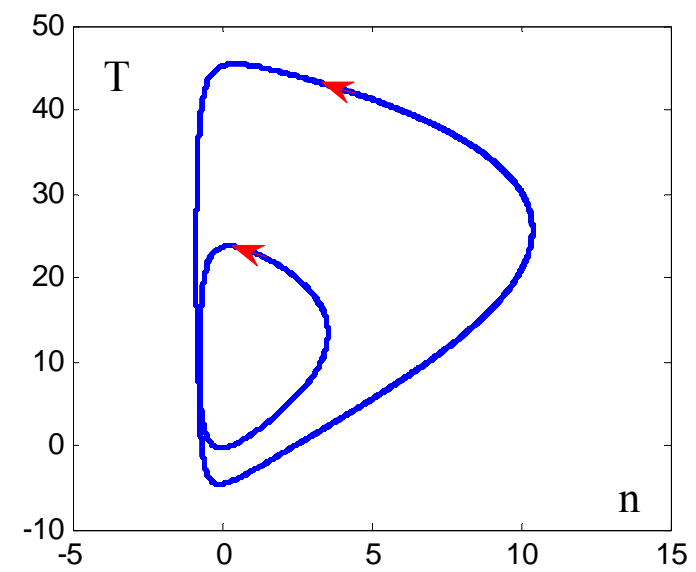

(c)

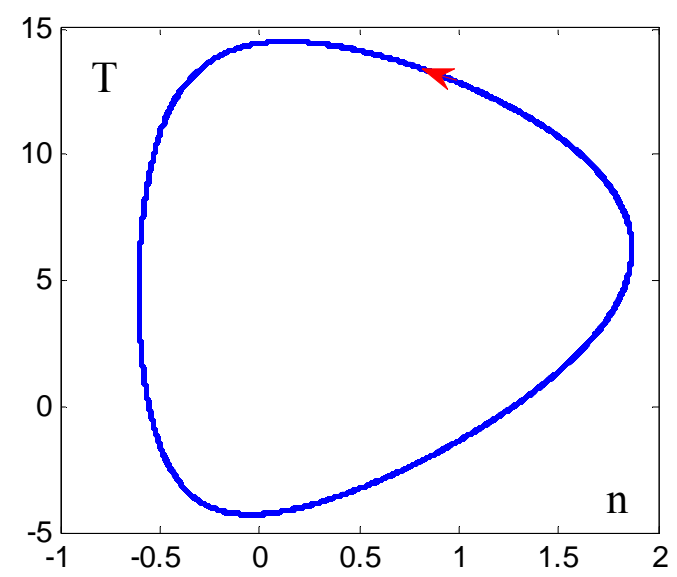

(b)

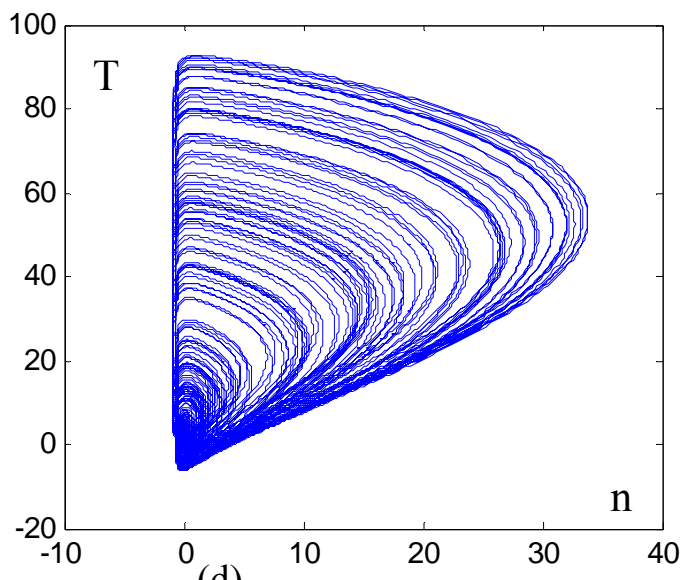


Figure 3
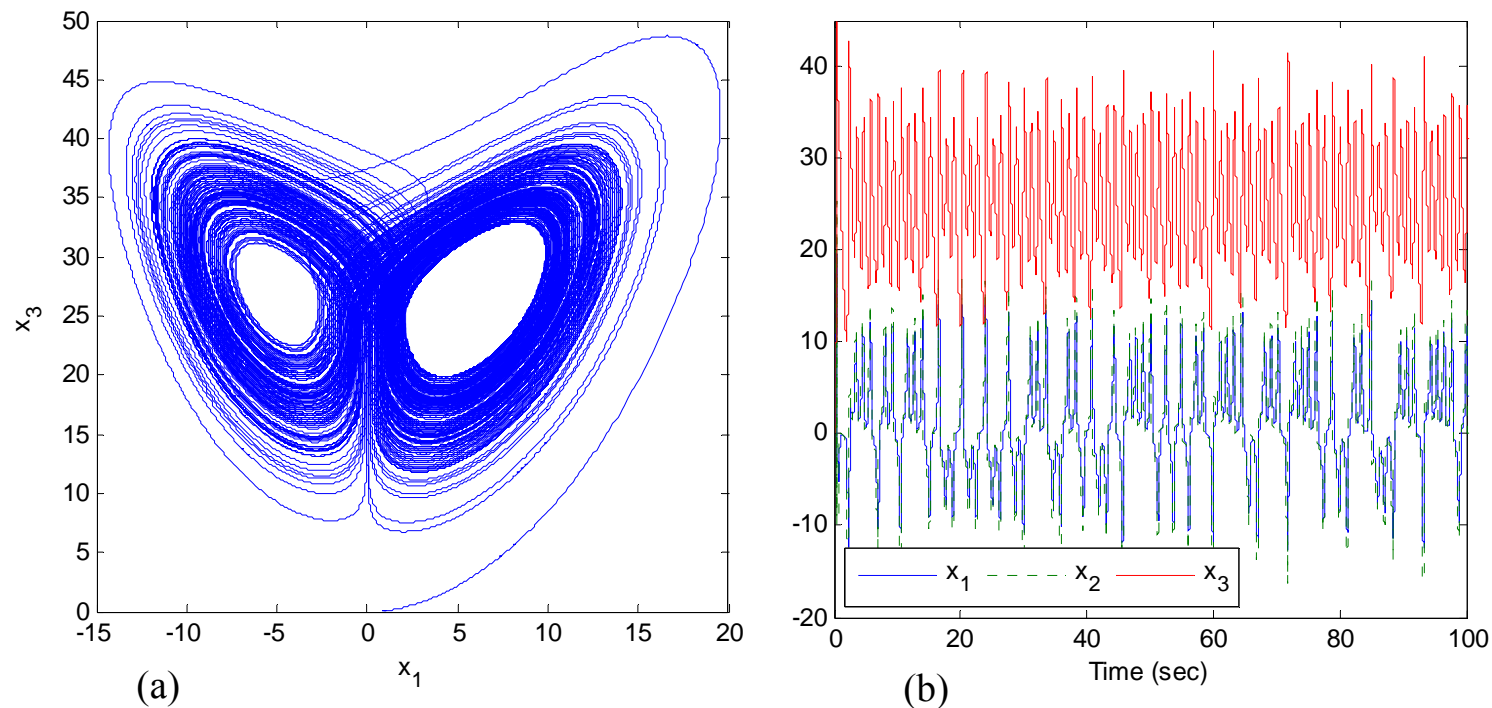
Figure 4
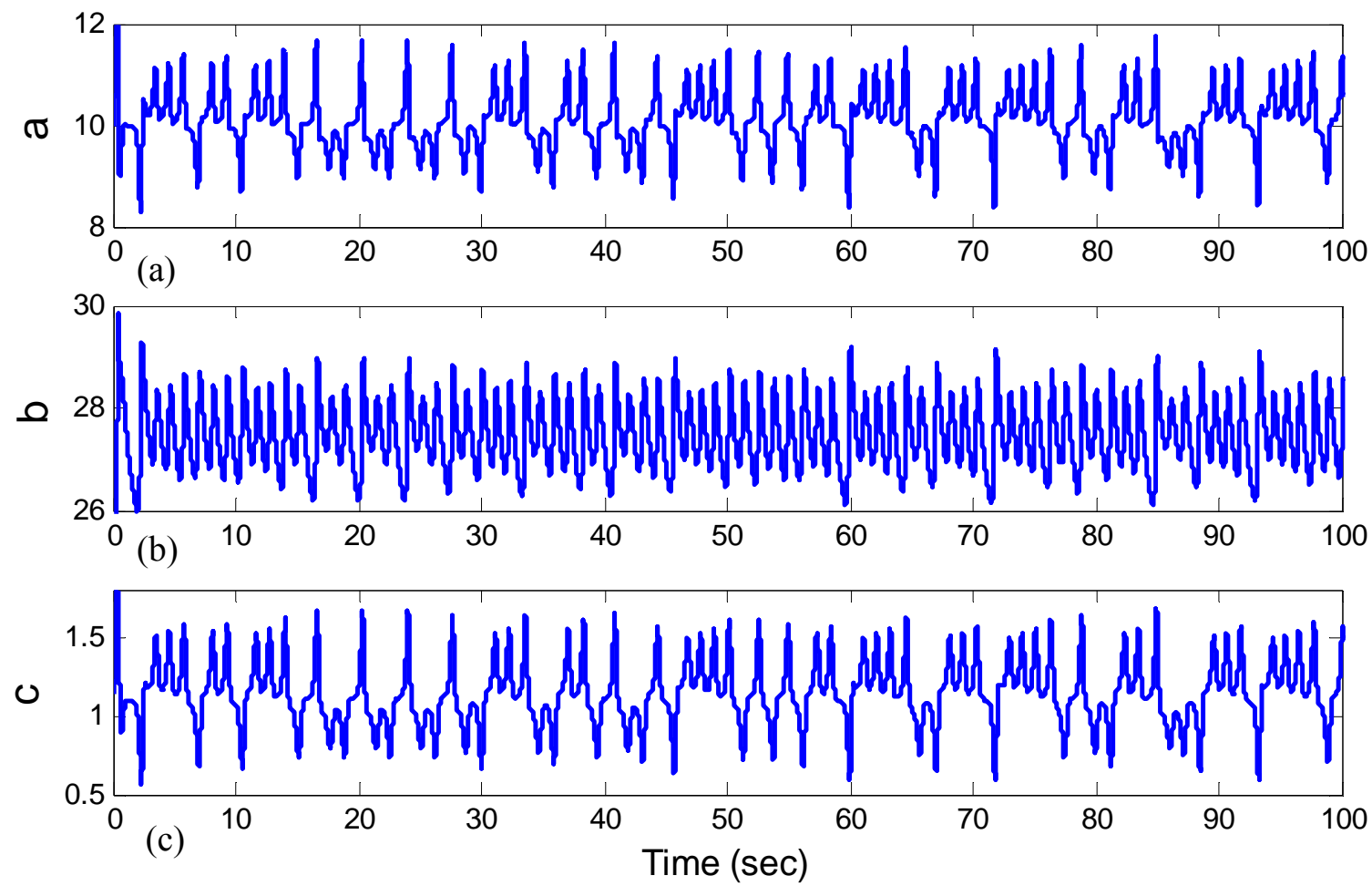
Figure 5

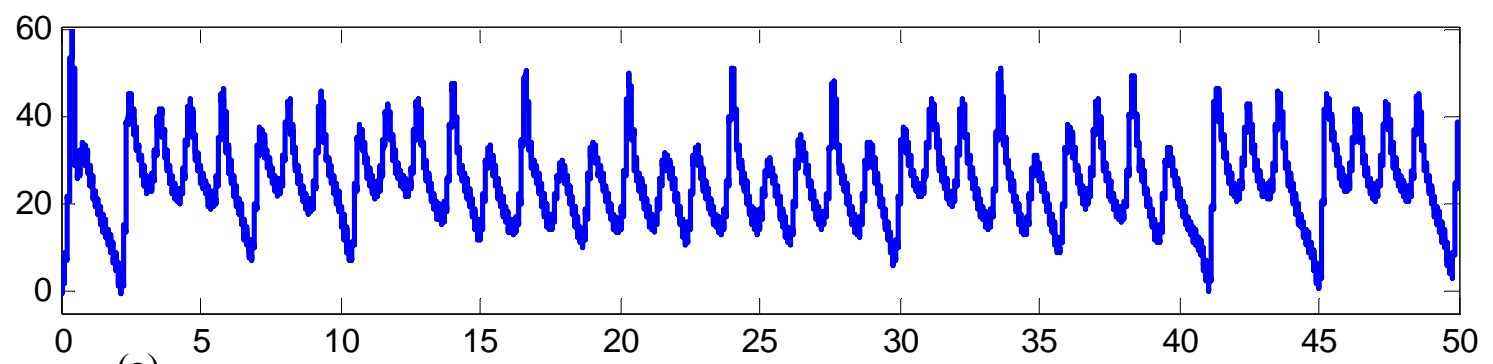

(a)
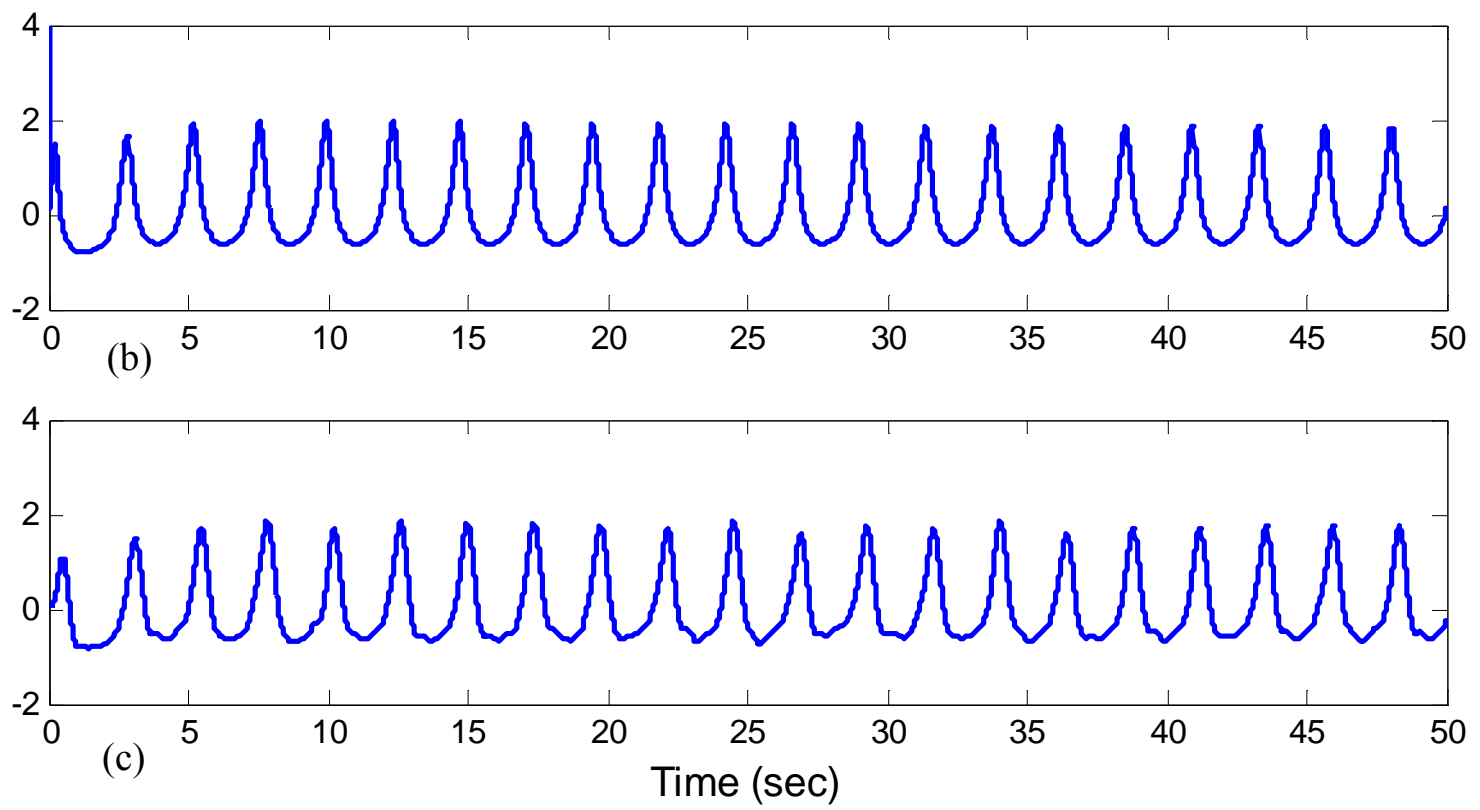
Figure 6
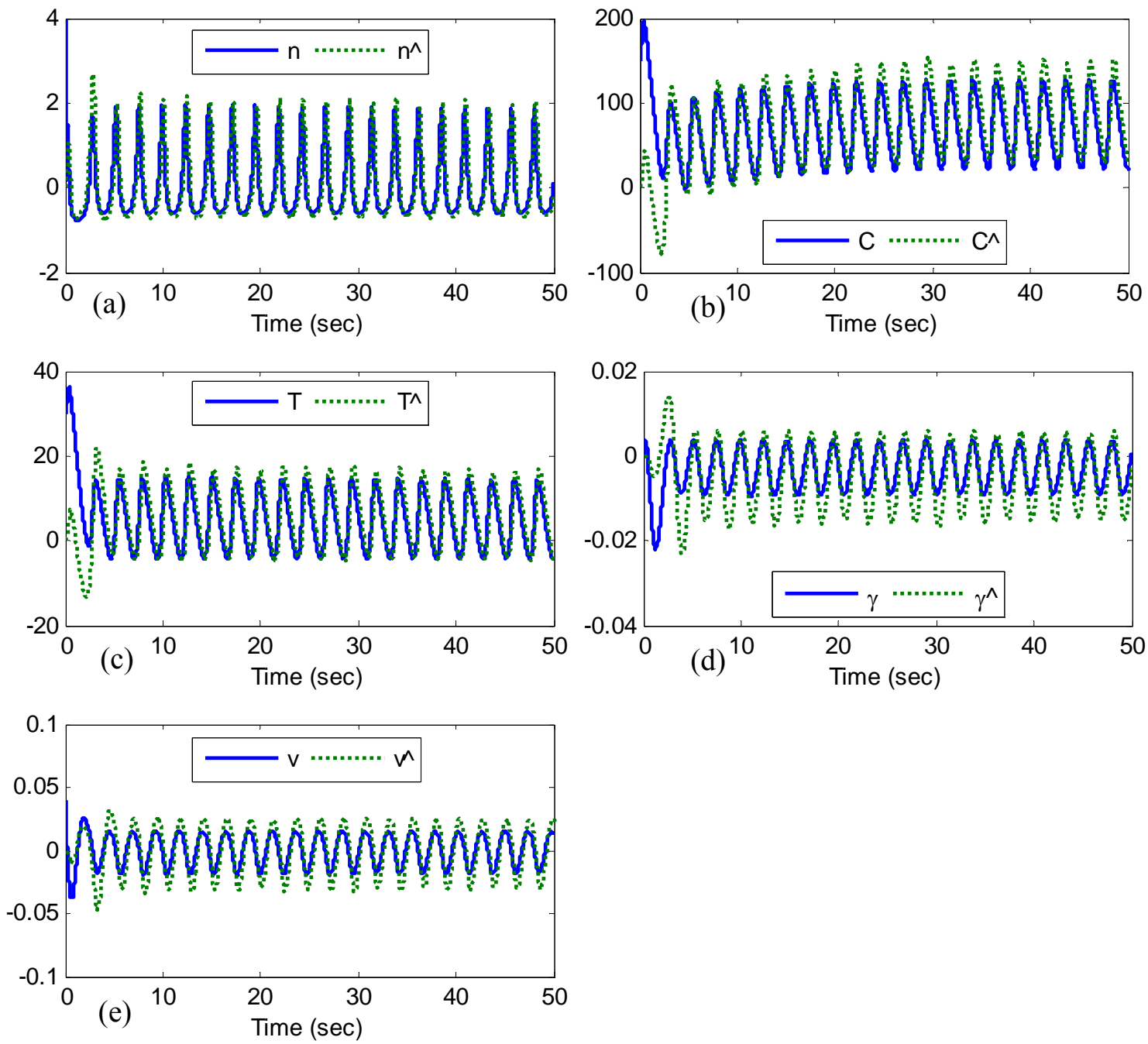
Figure 7

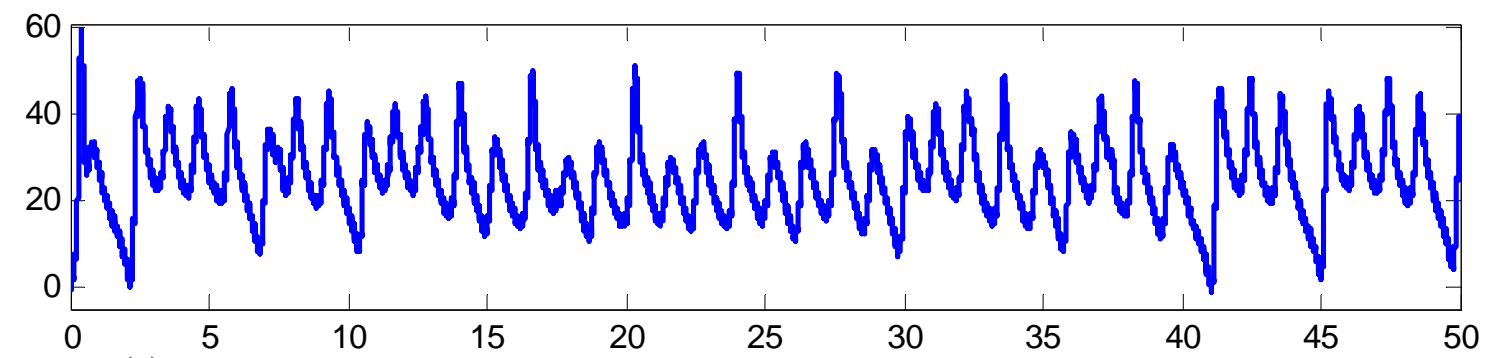

(a)

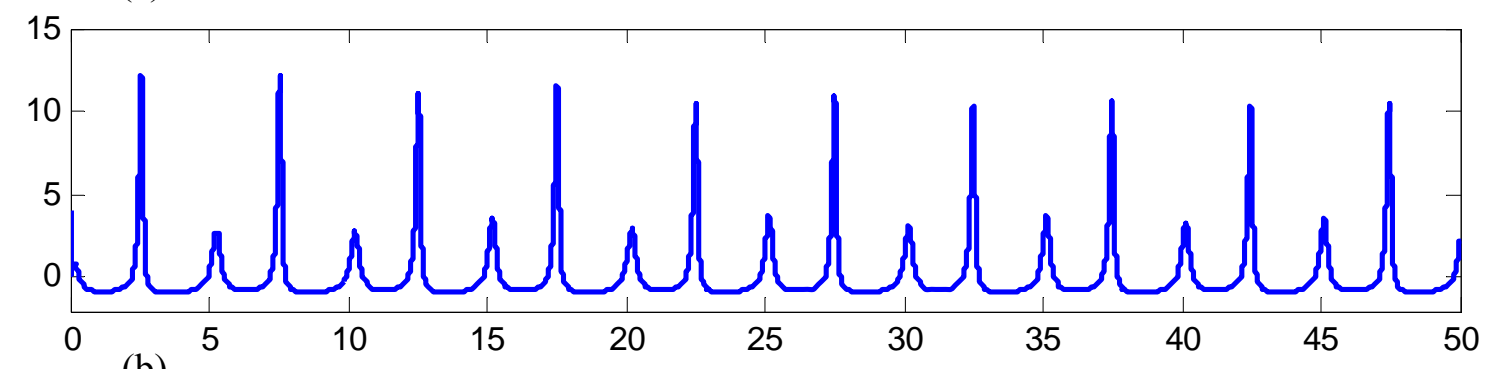

(b)

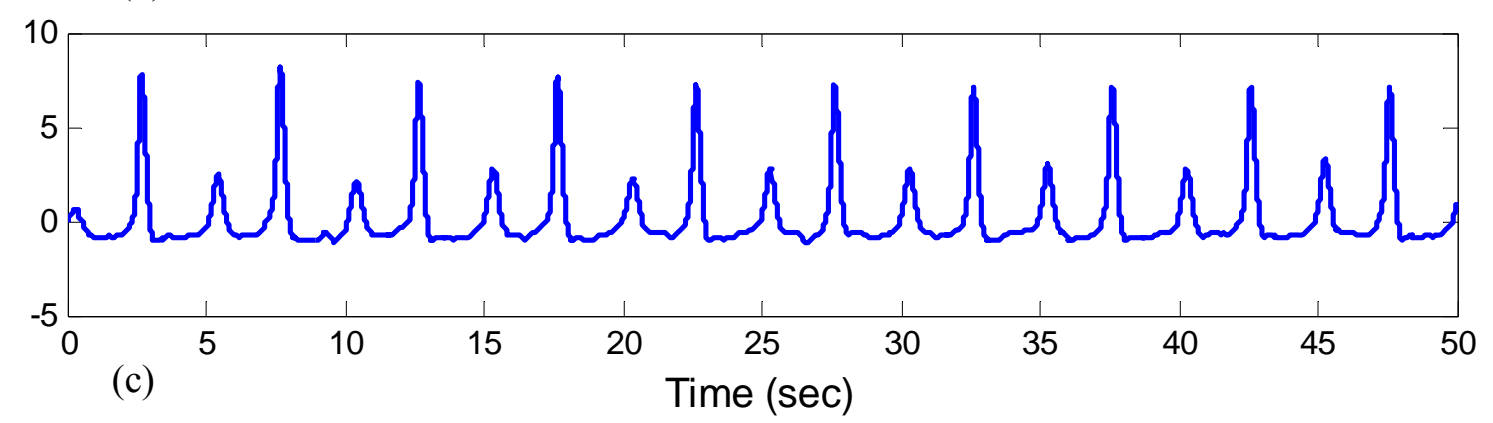


Figure 8
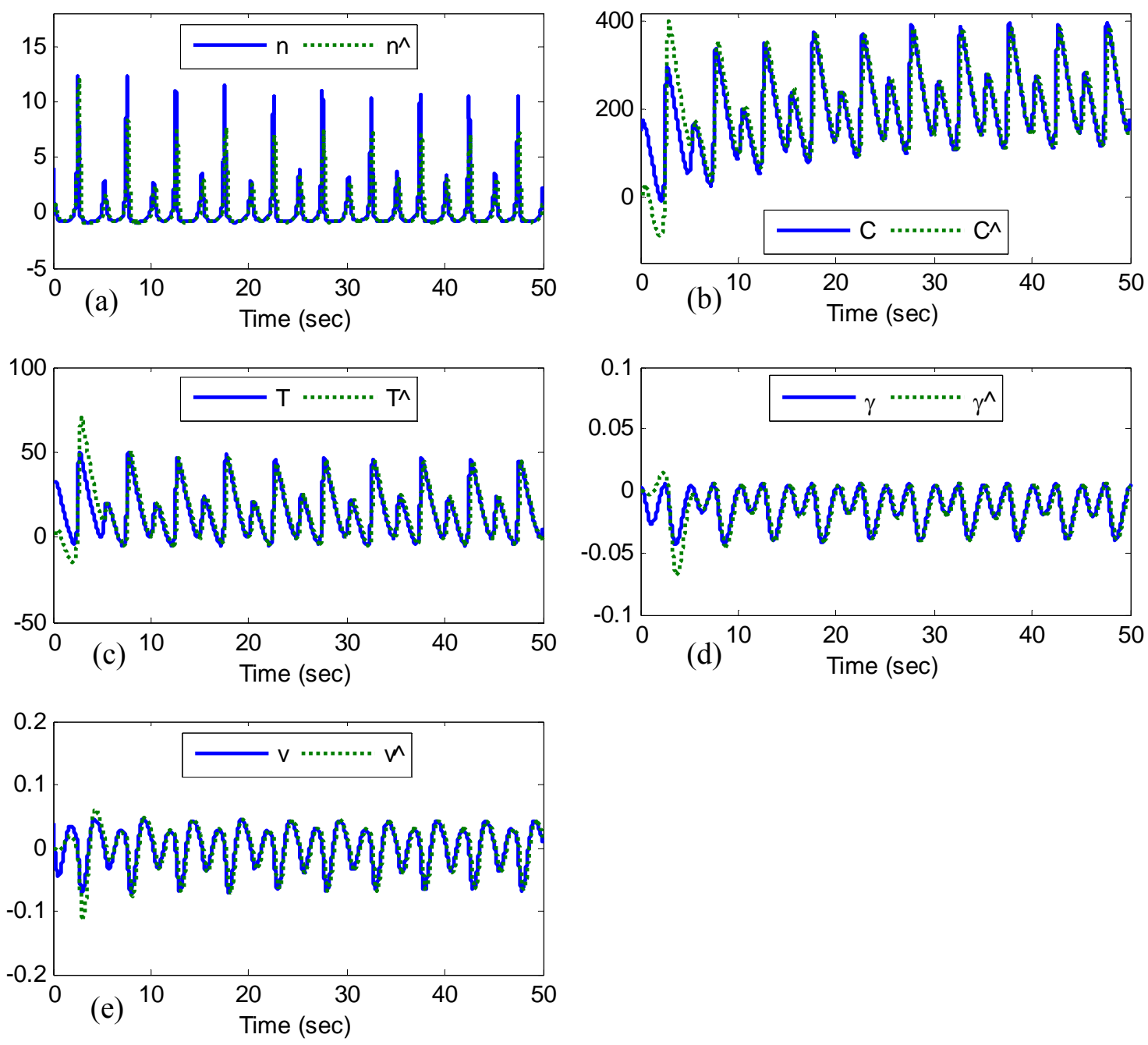
Figure 9
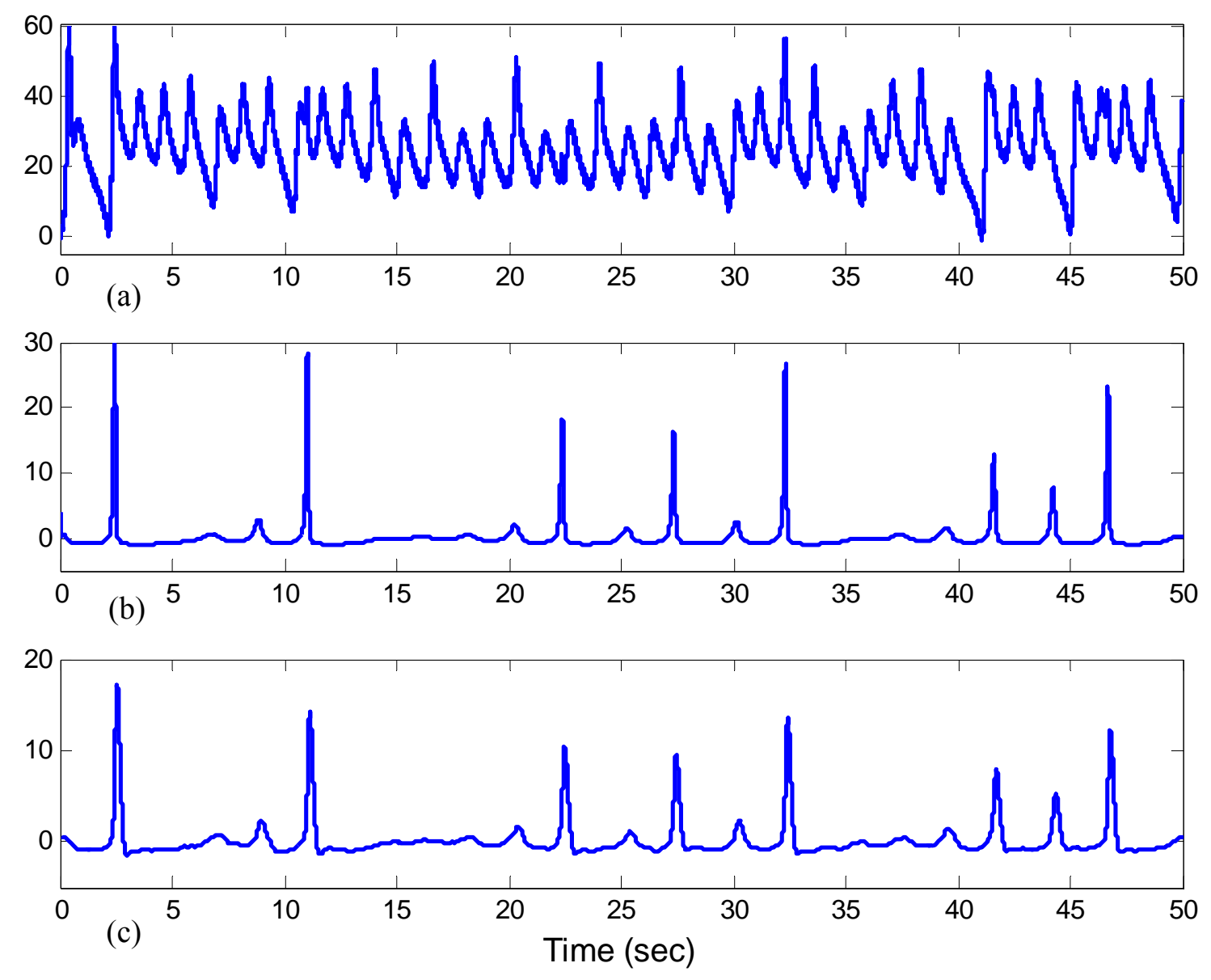
Figure 10
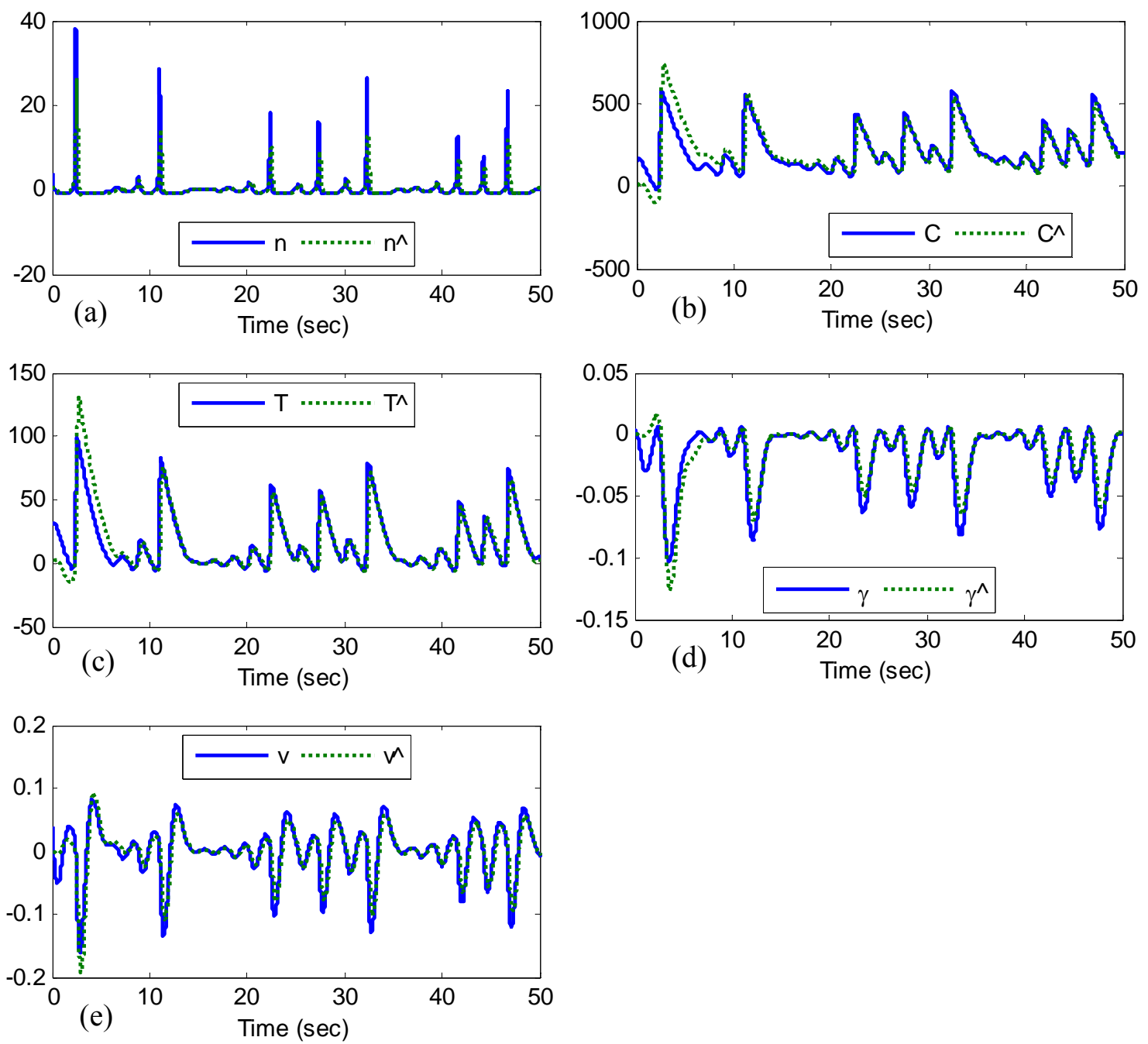
Figure 11
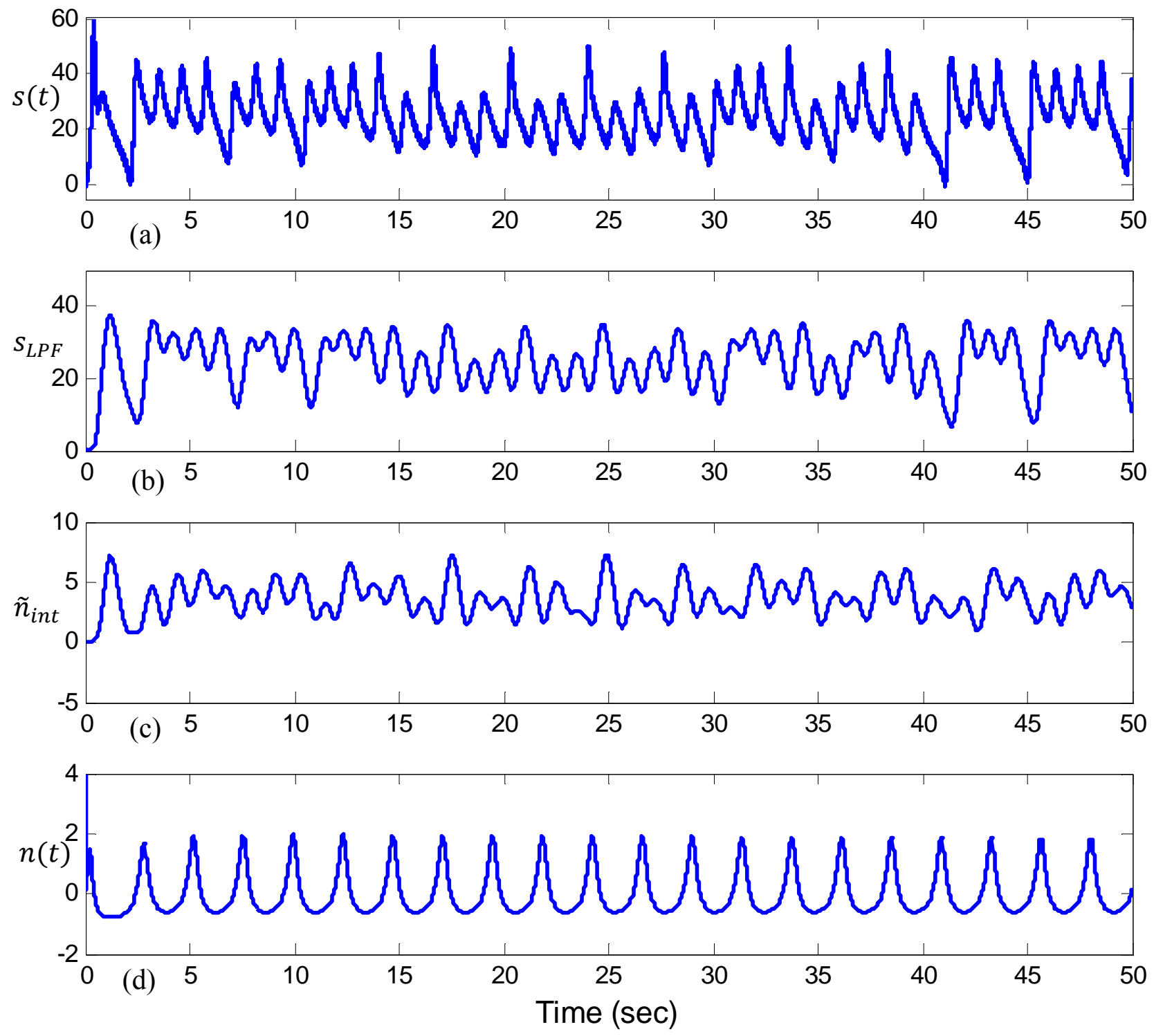